\title{
Laser-Induced-Forward-Transfer: A rapid prototyping tool for fabrication of photonic devices
}

\author{
S. Mailis, C. L. Sones, P. Ganguly, Y. J. Ying, K. S. Kaur, D. P. Banks, R.W. Eason \\ Optoelectronics Research Centre, University of Southampton, Highfield, Southampton, \\ SO17 1BJ, UK \\ e-mail: sm@orc.soton.ac.uk
}

Laser-Induced Forward Transfer (LIFT) [1] is a well established method for the spatially selective deposition of materials in a controllable dot-matrix printer fashion and offers a clear advantage over other methods for the micro-deposition of multilayered structures and organic materials.

Here we have used LIFT as a rapid prototyping tool for the fabrication of photonic circuits. More specifically we show that LIFT can be used for the fabrication of optical waveguides by providing a spatially selective metal source for thermal diffusion. Thus it is possible to combine the flexibility of a direct laser writing method with the large refractive index changes, low loss and stability that characterize in-diffused waveguides. LIFT provides the ability for easy fabrication of unconventional structures such as segmented $2 \mathrm{D}$ waveguides which provide a much higher degree of flexibility for tailoring the waveguide mode which can vary along the length of the waveguide. Additionally, LIFT allows co-doping with more than one element for the modification of other physical properties. Last, but not least, LIFT is a suitable method for depositing materials in non-planar geometries [2] such as fibres and ridges.

To illustrate the advantages of LIFT for rapid prototyping of photonic circuits we have fabricated waveguides by discrete deposition of Ti metal on lithium niobate $\left(\mathrm{LiNbO}_{3}\right)$ single crystal substrates followed by thermal diffusion. An ultrafast laser source $(150 \mathrm{fs}, 800 \mathrm{~nm})$ with a repetition rate of $1 \mathrm{kHz}$ was used for the deposition in the experimental arrangement which is described in [3]. Tailoring of the waveguide
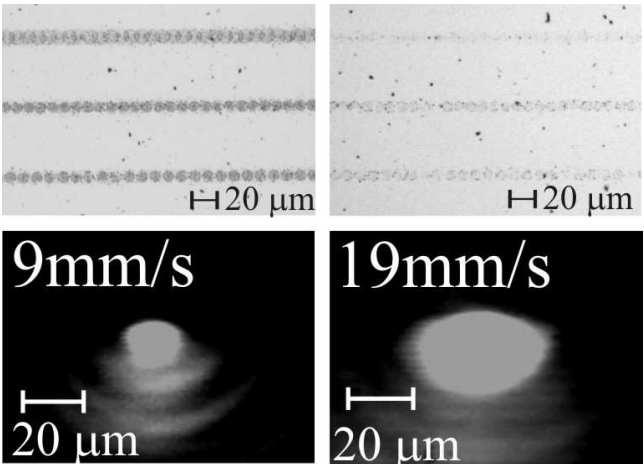

Figure1. Top left: Ti metal as deposited on the surface of $\mathrm{LiNbO}_{3}$. The scanning speed controls the separation between adjacent deposits Top right: the surface after thermal diffusion. Bottom: two waveguide mode profiles corresponding to different scanning speeds as indicated. mode profile was achieved by merely adjusting the deposition conditions (laser intensity, scanning speed) in a single deposition session. The mode profiles of two waveguides produced with different scanning speeds can be seen in the two bottom images shown in figure 1. Furthermore, the segmented nature of these waveguides resulted in a substantial extension of the, normally narrow, single mode spectral range showing the potential of this method to produce photonic structures with interesting spectral properties.

[1] J. Bohandy, B. F. Kim, and F. J. Adrian, J. Appl. Phys. 60, 1538(1986)

[2] S. Mailis, I. Zergioti, G. Koundourakis, A. Ikiades, A. Patentalaki, P. Papakonstantinou, N.A. Vainos and C. Fotakis, Appl. Opt. 38, 2301(1999)

[3] D. P. Banks, C. Grivas, J. D. Mills, R. W. Eason, and I. Zergioti Appl. Phys. Lett. 89, 193107 1-3 (2006) 